\title{
Clinico-radiological and bronchoscopic predictors of microbiological yield in sputum negative tuberculosis in Pakistan
}

\author{
Taymmia Ejaz¹, Mahmood Iqbal Malik², Jamal Ahmed², Rizwan Azam², Yousaf Jamal², Sheema Saadia' \\ ${ }^{1}$ Department of Medicine, Aga Khan University Hospital, Karachi; ${ }^{2}$ Department of Pulmonology, Pak Emirates \\ Military Hospital, National University of Medical Sciences, Rawalpindi, Pakistan
}

\begin{abstract}
To determine association of clinico-radiological factors and radiological activity with diagnostic yield in sputum-smear negative tuberculosis (TB). Prospective observational study in the Military Hospital Rawalpindi (Pakistan) from July to December 2018. Adult patients having no contraindications to bronchoscopy were included. HIV positive patients and those on anti-tuberculosis therapy for more than one week were excluded. High-resolution computed tomography (HRCT) findings were classified
\end{abstract}

Correspondence: Dr. Taymmia Ejaz, Department of Medicine, Aga Khan University Hospital, Stadium Road, Karachi, Pakistan. Tel. +92.3225830686 .

E-mail: taymmia.ejaz@gmail.com, taymmia.ejaz@aku.edu

Key words: Tuberculosis; HRCT; bronchoscopy; GeneXpert.

Contributions: TE, MIM, conceptualization, methodology; TE, YJ, SS, formal analysis; MIM, JA, RA, YJ, data curation; TE, SS, software; JA, YJ, validation; JA, RA, investigation; TE, MIM, SS, writing original draft preparation; JA, RA, YJ, SS, writing - review and editing. All the authors have read and approved the final version of the manuscript and agreed to be accountable for all aspects of the work

Conflict of interest: The authors declare that they have no competing interests, and all authors confirm accuracy.

Ethics approval and consent to participate: Ethical approval was taken from the Institutional Review Board. Informed consent was taken from patients prior to procedure.

Received for publication: 14 June 2021.

Accepted for publication: 5 October 2021.

Publisher's note: All claims expressed in this article are solely those of the authors and do not necessarily represent those of their affiliated organizations, or those of the publisher, the editors and the reviewers. Any product that may be evaluated in this article or claim that may be made by its manufacturer is not guaranteed or endorsed by the publisher.

${ }^{\circ}$ Copyright: the Author(s), 2021

Licensee PAGEPress, Italy

Monaldi Archives for Chest Disease 2022; 92:1976

doi: 10.4081/monaldi.2021.1976

This article is distributed under the terms of the Creative Commons Attribution-NonCommercial International License (CC BY-NC 4.0) which permits any noncommercial use, distribution, and reproduction in any medium, provided the original author(s) and source are credited. based on active and inactive tuberculosis features. Washings were sent for acid-fast bacillus (AFB) smear, GeneXpert assay and cultures. Out of 215 patients, $42.3 \%$ (91) were diagnosed with microbiological or histological evidence of TB. On univariate analysis, cavitation ( $p$-value $<0.001$ ), soft-tissue nodules (p-value 0.04 ), and endobronchial mucosal changes ( $p$-value 0.02 ) were associated with culture positivity. Presence of cavitation $(\mathrm{OR}=4.10 ; \mathrm{CI}=$ 2.18,7.73; p-value $<0.001$ ) was the only independent predictor of microbiological yield. Diagnostic yield was 70\%, 50\%, $12.5 \%$ and $8.6 \%$ in patients with definitely active, probably active, indeterminate and inactive tuberculosis HRCT features respectively. Sensitivity, specificity, positive predictive value and negative predictive value of HRCT active TB were $95.38 \%$ (95\% CI 87.1099.04), $48.00 \%$ (95\% CI 39.78-56.30), 44.29\% (95\% CI 40.31 $48.33), 96.00 \%(95 \% \mathrm{CI} 88.70-98.66)$ respectively. There was no significant association between age groups, smoking status and gender with diagnosis of tuberculosis in our study. Radiological activity and certain visualized bronchoscopic changes were associated with good diagnostic performance and can be used as predictive factors in diagnosis of active smear negative tuberculosis.

\section{Introduction}

Tuberculosis (TB) remains one of the leading causes of mortality and morbidity caused by an infectious agent [1]. TB caused approximately 2 million deaths and 11 million cases in 2018 worldwide [1]; while some 3.6 million people with TB remained undetected or were missed by health care systems every year and thus did not receive treatment [2]. Pakistan ranks $5^{\text {th }}$ among the 22 high burden countries which account for $80 \%$ of the cases worldwide, and also has the fourth highest burden of drug-resistant TB [1]. The end TB strategy 2035 target is a $90 \%$ reduction in TB incidence rate to less than $10 / 100,000$ as compared to 2015 [2], whereas smear negative TB often presents as a diagnostic challenge since only $60 \%$ of cases are smear positive on sputum. About one third, or an estimated 1.9 million cases of tuberculosis were sputum smear negative in 2011 [1,3]. Although it is associated with less mortality, and is considered less infectious, up to $20 \%$ tuberculosis transmission is attributed to sputum negative patients[4,5]. Patients with sputum-negative tuberculosis had relative transmissibility of 0.24 in study by Tostmann et al. [4] in Netherlands and 0.22 in study by Behr et al. [5]. Sputum culture yield is low and even with use of bronchoscopy and acquired specimen culture, diagnostic yield can vary from 30 to $90 \%$ [6], this together with to long incubation period required for culture testing can result in diagnostic delays. Therefore, a combination of clinical parameter, radiological activity and laboratory test such as interferon gamma release assay 
(IGRA) [7] can aid in improving diagnostic accuracy and management. Computer aided scoring based on clinical history features, chest X-ray findings together with microbiological testing increased diagnostic yield in a study done in over 6,845 presumptive tuberculosis patients in Pakistan [8].

Bronchoscopy aids in acquiring respiratory specimens, differentiating TB from other diseases and visualizing findings diagnostic of endobronchial tuberculosis. However, it is also over-utilized in certain cases. Cultures which are gold standard take weeks and culture positive rates vary from $12.5 \%-62.5 \%$ [9], due to various factors such as medium type ,respiratory specimen type such as bronchial washing and bronchoalveolar lavage (BAL). Computed tomography (CT) scan although more expensive, is more sensitive and specific than chest X-ray. This can aid in early diagnosis, treatment and prevent over diagnosis as incorrect treatment causes unnecessary burden on patients. High resolution CT (HRCT) is helpful in detecting indicators of active disease not seen on chest $\mathrm{X}$-ray, differentiating old from active case and identifying patients at higher risk of active TB. Important CT findings of active pulmonary tuberculosis are centrilobular nodules and branching linear structures (tree-in-bud appearance), lobular consolidation, cavitation, and bronchial wall thickening. The $\mathrm{CT}$ findings of inactive pulmonary tuberculosis include calcified nodules or consolidation, irregular linear opacity, parenchymal bands, and pericicatricial emphysema [10-12]. Smear-negative TB has a lower bacillary load and different clinical and radiological findings compared to smear positive $\mathrm{TB}$, therefore using criteria for smear-positive TB to predict risk in smear negative TB is not appropriate [13]. While studies on smear positive TB are many, studies that corelated culture results with CT scan features are few. CT scanning can help select patients for bronchoscopy and identify patients at higher risk of having active TB, however, findings on CT scan and bronchoscopy which are significantly associated with culture and microbiological yield vary in different studies and to our knowledge no such study specifically on smear negative TB has been done in Pakistan. This study was conducted with the aim of determining clinico-radiological and bronchoscopic findings which are associated with microbiological yield i.e., smear, GeneXpert and culture positivity and to determine factors associated with increased diagnostic yield in order to optimize and facilitate the appropriate use of investigations in correct clinical conditions.

\section{Patients and Methods}

This study was a prospective observational study carried out in Bronchoscopy unit of Pulmonology Department, Pak Emirates Military Hospital, Rawalpindi from July to December 2018. Ethical approval was taken from Institutional Review Board. Informed consent was taken from patients prior to procedure. Patients with sputum negative (both AFB smear and GeneXpert negative) tuberculosis or scare sputum who had not received empirical anti- tuberculosis therapy for more than one week, were included in the study. HIV positive patients, those having dual pathology, those who refused consent, patients younger than 18 years or having contraindications were excluded. Using non-probability consecutive sampling technique, patients fulfilling inclusion criteria were included in the study.

All patients underwent CT scan and bronchoscopy. HRCT was done on 128 slice Toshiba machine with $1.5 \mathrm{~mm}$ slice thickness. Where there was suspicion of malignancy, contrast enhanced CT chest (CECT) was carried out.
Based on another study protocol by Ko et al. [12], radiographic findings were classified into three groups on the basis of activity. Lesion including a cavity were classified as "definitely active"; treein-bud appearance or multiple non-calcified poorly circumscribed nodules without a cavity were classified as "probably active"; noncalcified well-circumscribed nodules as "indeterminate activity", and lesions appearing mainly as calcified nodules or fibrotic bands were classified as "probably inactive". In patients having two or more categories' lesions, higher category was selected.

Fiberoptic Bronchoscopy was carried out using $2.0 \mathrm{~mm}$ internal diameter flexible diagnostic bronchoscope. Abnormal findings on direct visualization during bronchoscopy were noted and appropriate endobronchial respiratory samples were taken. Endobronchial biopsy sample was taken in case of endobronchial mass. Samples were sent for AFB smear, GeneXpert assay, cultures and sensitivity while biopsy sample in case of mass were sent for histopathology.

Data were entered and analyzed in Statistical Package for Social Sciences (SPSS) version 23. Chi-square test was carried out for nominal/cateogorical data and percentages comparison. Sensitivity specificity, positive predictive value and negative predictive value for HRCT activity were calculated. The HosmerLemeshow goodness of fit test was done to assess model fit and logistical regression was done to determine CT findings associated with microbiological yield, $\mathrm{p}<0.05$ was considered significant.

\section{Results}

After exclusion of patients with incomplete data, HIV cases and dual pathology, a total of 215 patients were included in the study. Mean age was $48 \pm 18.18$ years. $67.4 \%$ were males and $32.6 \%$ were females. A total of $42.3 \%$ (91) were diagnosed with microbiological or histological evidence of TB, 30.2\% (65) had culture-confirmed MTB. The remaining $57.7 \%$ (124) cases had no evidence of tuberculosis on microbiology or histopathology after bronchoscopy.

Most common symptom reported by patients was cough $85.4 \%$ (181). However, none of the individual clinical symptoms correlated with increased microbiological yield. Combination of cough, fever and weight loss was seen in 14\% (30) and it was also not associated with a statistically significant culture yield $(\mathrm{p}=0.20)$. Clinical symptoms and bronchoscopy findings are summarized in Tables 1 and 2 .

Abnormal findings on bronchoscopic visualization were noted in $21.4 \%$ (46) (Table 2). Most common finding was presence of thick purulent secretions in $7.6 \%$ (16), followed by hyperemic mucosa in $6.3 \%$ (14). Endobronchial mass was seen in seven cases and $6 / 7$ patients with endobronchial growth were diagnosed with

Table 1. Clinical features of the study participants.

\begin{tabular}{lcc} 
Clinical findings & Frequency (n) & $\%$ \\
Cough & 181 & 85.40 \\
Fever & 90 & 42.50 \\
\hline Weight loss & 86 & 40.60 \\
Dyspnea & 45 & 21.20 \\
\hline Hemoptysis & 36 & 17.00 \\
Chest pain & 31 & 14.60 \\
\hline
\end{tabular}


TB, with culture positive-TB in five cases. Findings on bronchoscopy such as mucosal hyperemia were associated with increased likelihood of culture positivity, $64.3 \%$ of cases with hyperemia were culture positive. Overall mucosal changes (hyperemia, nodules, granulations, edema) were seen in $13.5 \%$ (29) cases and they were significantly associated with smear and culture positivity $(p=0.02)$. Mucosal changes on bronchoscopy were more commonly associated with presence of cavitation on HRCT $(\mathrm{p}<0.05)$.

On CT radiography, 41.4\% (89) had predominantly rightsided features, $17.2 \%$ (37) had left -sided and $41.4 \%$ (89) had bilateral changes. Most imaging abnormalities were seen in right apical segments, and the most common imaging finding was consolidation. All five cases of suspected miliary tuberculosis on radiology were negative for diagnostic evidence of tuberculosis. Only $34.5 \%$ $(10 / 29)$ of cases with tree-in-bud appearance were culture positive and overall, $44.8 \%(13 / 29)$ were diagnosed with tuberculosis. Among cases with pleural effusion, 46.2\% (6/13), 30.8\% (4/13) and $23.1 \%$ (3/13) were positive on GeneXpert, culture and AFB smear respectively. The only radiological finding significantly associated with tuberculosis diagnosis on univariate analysis was presence of cavitary changes or cavitary lesion with $\mathrm{p}<0.05$ for culture and GeneXpert positivity, as $66.2 \%$ (45/68) with cavitation had microbiological evidence of tuberculosis. Radiological findings summarized in Table 3.

Using the variables, tree-in-bud appearance, soft-tissue nodules, cavitation, consolidation and endobronchial mucosal changes on bronchoscopy, multivariable binary logistical regression was done (simultaneous entry method). Only the presence of cavitation $(\mathrm{OR}=4.10 ; \mathrm{CI}=2.18,7.73 ; \mathrm{p}<0.001)$ was found to be an independent predictor of microbiological yield (Table 4).

Patients were classified according to the radiographic activity on chest CT into $29.8 \%$ (64) definitely active group, 35.3\% (76) as the probably active group, $18.6 \%$ (40) as the indeterminate activity group, and $16.3 \%$ (35) into probably inactive group. MTB Culture was positive in $54.7 \%, 35.5 \%, 5 \%$ and $2.9 \%$ patients according to

Table 2. Bronchoscopy findings and microbiological yield.

\begin{tabular}{lccccc} 
& Frequency & $\%$ & Smear & GeneXpert & Culture \\
Normal & 169 & 78.6 & $35(20.7 \%)$ & $59(34.9 \%)$ & $46(27.2 \%)$ \\
Abnormal findings & 46 & 21.4 & $17(37 \%)$ & $21(45.7 \%)$ & $19(41.3 \%)$ \\
\hline p-value & & & 0.023 & 0.18 & 0.06 \\
Endobronchial mucosal changes* & 29 & 13.5 & $13(44.8 \%)$ & $14(48.3 \%)$ & $14(48.3)$ \\
\hline p-value & & & 0.005 & 0.08 & 0.002 \\
Secretions & 16 & 7.4 & $4(25 \%)$ & $7(43.8 \%)$ & $6(37.5 \%)$ \\
\hline Hyperemic mucosa & 14 & 6.5 & $8(57.1 \%)$ & $9(64.3 \%)$ & $9(64.3 \%)$ \\
Endobronchial growth & 7 & 3.3 & $5(71.4 \%)$ & $5(71.4 \%)$ & $5(71.4 \%)$ \\
\hline Nodular mucosa & 8 & 3.7 & $4(50 \%)$ & $3(37.5 \%)$ & $4(50 \%)$ \\
Extraluminal compression & 4 & 1.9 & $2(50 \%)$ & $2(50 \%)$ & $1(25 \%)$ \\
\hline Granulation & 3 & 1.4 & 0 & $1(33.3 \%)$ & 0 \\
VC palsy & 2 & 0.9 & $1(50 \%)$ & $1(50 \%)$ & $1(50 \%)$ \\
\hline Inflamed mucosa & 2 & 0.9 & $1(50 \%)$ & $1(50 \%)$ & $1(50 \%)$ \\
Tracheal distortion & 2 & 0.9 & 0 & $1(50 \%)$ & $0(50 \%)$ \\
\hline Stenosis/narrowing & 2 & 0.9 & $1(50 \%)$ & $1(50 \%)$ & $1(50 \%)$ \\
Infiltrated mucosa & 1 & 0.5 & $1(100 \%)$ & $1(100 \%)$ & $1(100 \%)$ \\
\hline
\end{tabular}

*Hyperemia mucosa, granulation, edema, nodules.

Table 3. Comparison of HRCT scan findings with microbiological yield.

\begin{tabular}{|c|c|c|c|c|c|c|c|}
\hline \multirow{2}{*}{ HRCT finding } & \multirow[t]{2}{*}{ Total } & \multicolumn{2}{|c|}{ GeneXpert } & \multirow[t]{2}{*}{ p-value } & \multicolumn{2}{|c|}{ Culture } & \multirow[t]{2}{*}{ p-value } \\
\hline & & Positive & Negative & & Positive & Negative & \\
\hline Consolidation & $94(43.7)$ & 39 (41.5) & $55(58.5)$ & 0.25 & $34(36.2)$ & $60(63.8)$ & 0.09 \\
\hline Soft-tissue attenuation nodules & $74(34.4)$ & $24(32.4)$ & $50(67.6)$ & 0.29 & $16(21.6)$ & $58(78.4)$ & $0.04^{*}$ \\
\hline Cavitary lesion & $68(31.6)$ & $39(57.4)$ & $29(42.6)$ & $<0.001^{*}$ & $34(50)$ & $34(50)$ & $<0.001^{*}$ \\
\hline Bronchiectasis & $41(19.1)$ & $11(26.8)$ & $3(73.2)$ & 0.12 & $10(24.4)$ & $31(75.6)$ & 0.36 \\
\hline Tree-in-bud & $29(13.5)$ & $12(41.4)$ & $17(58.6)$ & 0.61 & $10(34.5)$ & $19(65.5)$ & 0.59 \\
\hline Hilar/mediastinal lymphadenopathy & $17(7.9)$ & $2(11.8)$ & $15(88.2)$ & $0.024^{*}$ & $1(5.9)$ & $16(93.1)$ & $0.023^{*}$ \\
\hline Fibrotic bands / reticular shadows & $28(13.0)$ & $5(17.9)$ & $23(82.1)$ & $0.034^{*}$ & $3(10.7)$ & $25(89.3)$ & $0.016^{*}$ \\
\hline Pleural effusion & $13(6.0)$ & $6(46.2)$ & $7(53.8)$ & 0.55 & $4(30.8)$ & $9(69.2)$ & 1.00 \\
\hline Ground glass haze & $9(4.2)$ & $3(33.3)$ & $6(66.7)$ & 1.00 & $3(33.7)$ & $6(66.7)$ & 1.00 \\
\hline Hilar mass & $3(1.4)$ & $3(100)$ & 0 & $0.05^{*}$ & $3(100)$ & 0 & $0.027^{*}$ \\
\hline Miliary appearance & $5(2.3)$ & 0 & $5(100)$ & 0.16 & 0 & $5(100)$ & 0.326 \\
\hline
\end{tabular}


the radiographic activity on chest CT. With the inclusion of washings smear, GeneXpert and histopathological results, the diagnostic yield was increased to $70 \%, 50 \%, 12.5 \%$ and $8.6 \%$ respectively (Table 5). A total of $65.1 \%$ (140) patients had features of suggested active TB (definitely and probably active combined) and diagnostic yield was $59.3 \%$ in these patients.

Sensitivity, specificity, positive predictive value and negative predictive value when compared with culture in patients with suggested active TB was $95.38 \%$ (95\% CI 87.10-99.04), 48.00\% (95\% CI 39.78-56.30), 44.29\% (95\% CI 40.31-48.33), 96.00\% (95\% CI $88.70-98.66)$, respectively. There was no significant association of age groups, smoking status and gender with diagnosis of tuberculosis in our study.

\section{Discussion}

Most imaging abnormalities were seen in the right apical segments in various studies $[3,14]$, however most common imaging abnormalities varied in different studies. Consolidation in a study by Shin et al was the most common finding, in concordance with our study [15]; nodules were more common in study by Palud et al. [16]; tree-in-bud appearance in studies by Lee et al. and other $[10,17]$. In agreement with our study, cavitation was associated with GeneXpert positivity in a study by King Wang et al. [18], their study also found no association of GeneXpert between presence or absence of tree-in-bud appearance and consolidation. Similarly, Shimon et al. [19] found microbiological evidence of MTB in only $3.7 \%(12 / 326)$ of patients with tree-in-bud appearance and concluded that the finding does not imply a specific pathogen. Desai et al. [20] found no association with the tree-inbud finding and their yield was higher in patients with co-existing consolidation or cavitation and in agreement with our study, presence of cavitation on $\mathrm{CT}$ was also the only predictor of a signifi- cant microbiological yield in their study. However, contrary to our study, tree-in-bud appearance was significantly associated with active TB in studies by Shin et al. [15], Nakanishi et al. [21], and Raghuvanshi et al. [22] and they also did not find any significant association with cavitation. Tayfun et al. [14] found no differences in HRCT findings in patients based on culture status.

Bronchoscopy findings were also in agreement with other studies in which most bronchoscopies were unremarkable, only 6 $(15 \%)$ patients had mucosal changes suggestive of tuberculosis in one study [23]. Similarly, findings were also normal in $40.6 \%$ patients in one other study by Shankar et al. in India [24]. However, in one study, a significantly higher $37.96 \%$ of cases showed mucosal congestion and hyperemia, $14.81 \%$ of patients had erosions and ulcerations as compared to the $13 \%$ in our study [25]; 60\% had mucosal hyperemia in study by Ritesh et al. [26] . These were associated with cavitation on HRCT in study by Choudary et al. [25], in agreement with our findings as mucosal changes on bronchoscopy were significantly associated with presence of cavitary changes on HRCT $(\mathrm{p}<0.05)$. In a study by Ozkaya et al. [27], BAL fluid smear AFB was positive in $26 \%$ and cultures in $39.1 \%$ cases with features of endobronchial tuberculosis as compared to $64.3 \%$ culture positive cases in our study. However, in contrast, higher BAL smear and culture yields of $62.5 \%$ and $93.7 \%$ were reported by Şimşek et al. [28] in Turkey. Although endobronchial TB is more common in females [27-29] we did not find any such association as, $62.1 \%$ of these mucosal changes were found in men in our study.

The diagnostic performance with regards to sensitivity and NPV of HRCT were similar to that of Ko et al. [12] and a lower specificity was also reported by them. In our study, sensitivity $95.38 \%$ was higher than the $80 \%$ reported by Lee et al. [30]; however, specificity of $45 \%$ in our study was lower than the $71 \%$ reported by them. In a systematic review of 13 studies utilizing radiographic features in TB scoring by Pinto et al. [31], presence of upper lobe infiltrates and cavities was significantly associated

Table 4. Logistical regression analysis to determine association with microbiological yield.

\begin{tabular}{lccccccc} 
Variables & Coefficient $(\boldsymbol{\beta})$ Standard error & Wald & Odds ratio & $\begin{array}{c}95 \% \text { confidence } \\
\text { interval }\end{array}$ & $\begin{array}{c}\text { P-value } \\
\text { Tree-in-bud }\end{array}$ \\
Soft-tissue nodules & 0.34 & 0.46 & 0.54 & 1.40 & $0.56-3.49$ & 0.461 \\
\hline Cavitation & -0.34 & 0.35 & 0.94 & 0.71 & $0.35-1.41$ & 0.33 \\
Consolidation & 1.41 & .32 & 19.11 & 4.10 & $2.18-7.73$ & 0.000 & 0.524 \\
\hline Endobronchial mucosal changes & 0.20 & 0.31 & 0.40 & 1.22 & $0.66-2.27$ & $0.69-3.85$ & 0.265 \\
\hline
\end{tabular}

Table 5. Diagnostic yield according to radiographic activity.

\begin{tabular}{|c|c|c|c|c|c|c|c|}
\hline \multirow[t]{2}{*}{ Radiographic activity } & \multirow[t]{2}{*}{ Total \% (n) } & \multicolumn{4}{|c|}{ Diagnostic yield } & \multirow[t]{2}{*}{ Non-diagnostic } & \multirow[t]{2}{*}{ p-value } \\
\hline & & $\begin{array}{l}\text { GeneXpert } \\
\text { positive }\end{array}$ & $\begin{array}{l}\text { Culture } \\
\text { positive }\end{array}$ & $\begin{array}{c}\text { AFB } \\
\text { positive }\end{array}$ & Total & & \\
\hline Suggested active & $65.1(140)$ & $52.9(74)$ & $44.30(62)$ & $35.70(50)$ & $59.3(83)$ & $40.7(57)$ & $<0.001$ \\
\hline Definitely active & $29.8(64)$ & $60.9(39)$ & $54.70(35)$ & $43.80(28)$ & $70.3(45)$ & $29.7(19)$ & \\
\hline Probably active & $35.3(76)$ & $46.1(35)$ & 35.50 (27) & $28.90(22)$ & $50.0(38)$ & $50(38)$ & \\
\hline Suggested inactive & $34.9(75)$ & $8(6)$ & $4(3)$ & $2.7(2)$ & $10.7(8)$ & $89.3(67)$ & $<0.001$ \\
\hline Indeterminate & $18.6(40)$ & $10.0(4)$ & $5.00(2)$ & $5.00(2)$ & $12.5(5)$ & $87.5(35)$ & \\
\hline Inactive & $16.3(35)$ & $5.7(2)$ & $2.90(1)$ & 0.00 & $8.6(3)$ & $91.4(32)$ & \\
\hline
\end{tabular}

Suggested active, definitely and probably active; suggested inactive, indeterminate and inactive; total diagnostic yield, including smear, GeneXpert, culture and histopathology results; AFB, acid fast bacillus. 
with TB, which is also in agreement with our findings. Sensitivity and specificity of "highly suspected pulmonary TB" findings on CT were found to be $95 \%$ and $40 \%$ respectively by Alsoway et al. [3], $90 \%$ and $50 \%$ by Shaarraway et al. [13], $100 \%$ and $30 \%$ by Nakanishi et al. [21]. Rank I HRCT criteria of highly suspected TB in these studies was defined as the Presence of at least three of; main lesion in S1, S2 or S6 segments, tree in bud appearance. lobular consolidations or larger nodules. Tozkoparan et al. [32] also found good diagnostic accuracy of HRCT with sensitivity, specificity, positive predictive value and negative predictive value of $88 \%, 88 \%, 92 \%, 83 \%$, respectively. Although, HRCT alone had good sensitivity in most of these studies and our study, the low PPV and specificity can hamper decisions to start ATT [30].

The potential limitations of our study include selection bias as certain patients in whom bronchoscopy was contraindicated were excluded and missed. Cost also precluded all patients from undergoing bronchoscopy as not everyone can afford CT and bronchoscopy in resource- poor settings. CT imaging protocol was not uniform due to different machines used and radiologists' reporting blinding was not done. Immunocompromised patients were also excluded. Some studies devised scoring systems by inclusion of clinical parameters, however, as stated by Tozkoparan et al. [32] factors, such as TB burden, clinical spectrum of the disease, physician experience, empirical treatment and medical history can confound these prediction models and thus these models have different yield in various clinical settings.

\section{Conclusions}

Although clinical parameters did not differ, significant differences were observed in the HRCT findings of smear-negative culture-positive and smear-negative culture-negative TB patients. The microbiological yield was higher in patients with HRCT scan cavitary changes and mucosal changes on bronchoscopy. Overall, microbiological diagnostic yield from specimen obtained by bronchoscopy varied based on radiological activity. The diagnostic yield was significantly higher $(70.3 \%)$ in patients with active radiological features and only $12.5 \%$ and $9.6 \%$ in patients with indeterminate activity and probably inactive radiological features. Mucosal changes on bronchoscopy were associated with smear and culture positivity and were more common in patients with cavitation on HRCT scan. HRCT has good diagnostic value in detecting activity of sputum smear-negative TB and predicting patients at high risk of active TB. HRCT can be especially useful in patients in whom bronchoscopy is not possible or have unequivocal findings due to empirical TB treatment. However, due to low specificity and non-confirmatory nature of HRCT, clinicians should also consider other diagnostic possibilities and diagnosis should be based on microbiological evidence where possible. Flexible bronchoscopy is an excellent diagnostic tool with appropriate microbiological yield in sputum negative tuberculosis which is particularly important in areas with high prevalence of multi-drug resistant tuberculosis.

\section{References}

1. World Health Organization. Global Tuberculosis Report 2018. Available from: https://apps.who.int/iris/handle/10665/274453
2. Raviglione M, Sulis G. Tuberculosis 2015: burden, challenges and strategy for control and elimination. Infect Dis Rep 2016;8:33-7.

3. Alsowey AM, Amin MI, Said AM. The predictive value of multidetector high resolution computed tomography in evaluation of suspected sputum smear negative active pulmonary tuberculosis in Egyptian Zagazig University Hospital Patients. Polish J Radiol 2017;82:808-16.

4. Tostmann A, Kik S V., Kalisvaart NA, et al. Tuberculosis transmission by patients with smear-negative pulmonary tuberculosis in a large cohort in the Netherlands. Clin Infect Dis 2008;47:1135-42.

5. Behr MA, Warren SA, Salamon H, et al. Transmission of Mycobacterium tuberculosis from patients smear-negative for acid-fast bacilli. Lancet 1999;353:444-9.

6. Prasad R, Singh A. Role of bronchoscopy in diagnosis of smear-negative pulmonary tuberculosis. Egypt J Bronchol 2019;131-5.

7. Ji L, Lou YL, Wu ZX, et al. Usefulness of interferon- $\gamma$ release assay for the diagnosis of sputum smear-negative pulmonary and extra-pulmonary TB in Zhejiang Province, China. Infect Dis Poverty 2017;6:1-5.

8. Zaidi SMA, Habib SS, Van Ginneken B, et al. Evaluation of the diagnostic accuracy of Computer-aided detection of tuberculosis on chest radiography among private sector patients in Pakistan. Sci Rep 2018;8:1-9.

9. Mondoni M, Repossi A, Carlucci P, et al. Bronchoscopic techniques in the management of patients with tuberculosis. Int $\mathrm{J}$ Infect Dis 2017;64:27-37.

10. Majmudar DK, Rajput DK. Role of HRCT in diagnosing disease activity of pulmonary tuberculosis. Int J Contemp Med Res 2017;4:1724-7.

11. Bhalla A, Goyal A, Guleria R, et al. Chest tuberculosis: Radiological review and imaging recommendations. Indian $\mathrm{J}$ Radiol Imaging 2015;25:213.

12. Ko Y, Lee HY, Park YB, et al. Correlation of microbiological yield with radiographic activity on chest computed tomography in cases of suspected pulmonary tuberculosis. PLoS One 2018;13:e0201748.

13. Shaarrawy H, Zeidan M, Nasr A, et al. Assessment of the role of high resolution computed tomography in the diagnosis of suspected sputum smear negative active pulmonary TB. Egypt J Chest Dis Tuberc 2013;62:263-8.

14. Çalişkan T, Ozkisa T, Aribal S, et al. High resolution computed tomography findings in smear-negative pulmonary tuberculosis patients according to their culture status. J Thorac Dis 2014;6:706-12.

15. Shin JA, Chang YS, Kim TH, et al. Fiberoptic bronchoscopy for the rapid diagnosis of smear-negative pulmonary tuberculosis. BMC Infect Dis 2012;12:141.

16. Le Palud P, Cattoir V, Malbruny B, et al. Retrospective observational study of diagnostic accuracy of the Xpert ${ }^{\circledR}$ MTB/RIF assay on fiberoptic bronchoscopy sampling for early diagnosis of smear-negative or sputum-scarce patients with suspected tuberculosis. BMC Pulm Med 2014;14:137.

17. Lee JY, Lee KS, Jung KJ, et al. Pulmonary tuberculosis: CT and pathologic correlation. J Comput Assist Tomogr 2000;24:691-8.

18. To KW, Kam KM, Chan DPC, et al. Utility of GeneXpert in analysis of bronchoalveolar lavage samples from patients with suspected tuberculosis in an intermediate-burden setting. J Infect 2018;77:296-301.

19. Shimon G, Yonit W-W, Gabriel I, et al. The "tree-in-bud" pat- 
tern on chest CT: Radiologic and microbiologic correlation. Lung 2015;193:823-9.

20. Desai SR, Edey AJ, Hansell DM, et al. Morphologic predictors of a microbiological yield in patients with a tree-in-bud pattern on computed tomography. J Thorac Imaging 2014;29:240-5.

21. Nakanishi M, Demura Y, Ameshima S, et al. Utility of highresolution computed tomography for predicting risk of sputum smear-negative pulmonary tuberculosis. Eur J Radiol 2010;73:545-50.

22. Raghuvanshi V, Gopal Sood R, Jhobta A, et al. Use of high-resolution computed tomography (HRCT) in diagnosis of sputum negative pulmonary tuberculosis. Turkish Thorac J 2016;17:59-64.

23. Haque S, Quaiser S, Agarwal A, et al. Fiberoptic bronchoscopy, as a valuable diagnostic option in sputum negative pulmonary tuberculosis: A prospective study. Int J Appl Basic Med Res 2012;2:123.

24. Shankar M, Saha KK, Sneha K, et al. Utility of fibreoptic bronchoscopy: An experience from a tertiary health care centre of Bihar, India. Int J Contemp Med Res 2018;5:1-4.

25. S C, Bo T, S K, et al. Outcome of fiber optic bronchoscopy in sputum smear negative pulmonary tuberculosis. Panacea $\mathrm{J}$ Med Sci 2015;5:33-9.

26. Sharma R, Sahasrabuddhe T, Showkat M, et al. A prospective study to evaluate the utility of bronchoalveolar lavage by fiberoptic bronchoscopy in sputum smear negative patients with high suspicion of pulmonary tuberculosis. Med J Dr DY Patil Univ 2012;5:43.

27. Ozkaya S, Bilgin S, Findik S, et al. Endobronchial tuberculosis: histopathological subsets and microbiological results. Multidiscip Respir Med 2012;7:34.

28. Şimşek A, Yapıcı İ, Babalık M, et al. Bronchoscopic diagnostic procedures and microbiological examinations in proving endobronchial tuberculosis. J Bras Pneumol 2016;42:191-5.

29. Peposhi IP, Kapisyzi PQ, Hafizi HS, et al. Value of bronchoscopy in the diagnosis of sputum smear negative pulmonary tuberculosis. Int J Res Med Sci 2017;5:3393

30. Lee J-J, Chong P-Y, Lin C-B, et al. High resolution chest CT in patients with pulmonary tuberculosis: Characteristic findings before and after antituberculous therapy. Eur J Radiol 2008;67:100-4.

31. Pinto LM, Pai M, Dheda K, et al. Scoring systems using chest radiographic features for the diagnosis of pulmonary tuberculosis in adults: a systematic review. Eur Respir J 2013;42:480-94.

32. Tozkoparan E, Deniz O, Ciftci F, et al. The roles of HRCT and clinical parameters in assessing activity of suspected smear negative pulmonary tuberculosis. Arch Med Res 2005;36:166-70. 